\title{
Aptamer-Mediated Delivery of Splice-Switching Oligonucleotides to the Nuclei of Cancer Cells
}

\author{
Jonathan W. Kotula, ${ }^{1}$ Elizabeth D. Pratico, ${ }^{1}$ Xin Ming, ${ }^{2}$ Osamu Nakagawa, ${ }^{2}$ \\ Rudolph L. Juliano, ${ }^{2}$ and Bruce A. Sullenger ${ }^{1}$
}

To reduce the adverse effects of cancer therapies and increase their efficacy, new delivery agents that specifically target cancer cells are needed. We and others have shown that aptamers can selectively deliver therapeutic oligonucleotides to the endosome and cytoplasm of cancer cells that express a particular cell surface receptor. Identifying a single aptamer that can internalize into many different cancer cell-types would increase the utility of aptamer-mediated delivery of therapeutic agents. We investigated the ability of the nucleolin aptamer (AS1411) to internalize into multiple cancer cell types and observed that it internalizes into a wide variety of cancer cells and migrates to the nucleus. To determine if the aptamer could be utilized to deliver therapeutic oligonucleotides to modulate events in the nucleus, we evaluated the ability of the aptamer to deliver spliceswitching oligonucleotides. We observed that aptamer-splice-switching oligonucleotide chimeras can alter splicing in the nuclei of treated cells and are effective at lower doses than the splice switching oligonucleotides alone. Our results suggest that aptamers can be utilized to deliver oligonucleotides to the nucleus of a wide variety of cancer cells to modulate nuclear events such as RNA splicing.

\section{Introduction}

$\mathbf{S}$ TANDARD CANCER THERAPIES SUCH AS chemotherapeutics and radiation are not only toxic to tumor cells but to healthy cells as well, which causes toxicity to the patient. This toxicity adversely affects patients, creating many serious conditions such as gastrointestinal distress, organ damage, and death. To attempt to reduce the adverse effects of cancer therapy and concurrently increase their efficacy, new therapeutics that specifically target cancer cells are needed. Aptamer-mediated delivery of therapeutic agents to targeted cells represents an emerging strategy that may be useful in treating cancer patients (Chu et al., 2006a; Chu et al., 2006b; Farokhzad et al., 2006; McNamara et al., 2006; Wullner et al., 2008; Dassie et al., 2009; Zhou and Rossi, 2010; Min et al., 2011; Wu et al., 2011).

Aptamers that recognize cell surface receptors have been utilized to deliver various cargos including toxins and small interfering RNA (siRNA) (Chu et al., 2006a; Chu et al., 2006b; Farokhzad et al., 2006; McNamara et al., 2006; Wullner et al., 2008; Dassie et al., 2009; Tiemann and Rossi, 2009; Kim et al., 2010; Li et al., 2010; Zhou and Rossi, 2010). Aptameroligonucleotide chimeras may be particularly safe for treating cancer because they can be engineered to contain 2 layers of selectivity: (a) an aptamer domain that binds to a receptor overexpressed on cancer cells and (b) a therapeutic oligonucleotide that affects an essential pathway in cancer but not normal cells. For example, we and others have described the use of an aptamer targeting prostate specific membrane antigen (PSMA) to deliver siRNAs to inhibit the expression of genes that are upregulated in prostate cancer cells such as polo-like kinase 1 (Plk1), elongation factor2 (EEF2), and B-cell lymphoma-extra large (Bcl-xL) (McNamara et al., 2006; Wullner et al., 2008; Dassie et al., 2009; Kim et al., 2010). This double layer of specificity has the potential to dramatically increase the selectivity of therapeutics, which may greatly reduce their side effects.

Recently, our lab and others have generated aptamers that recognize a limited number of other cell surface receptors (Dollins et al., 2008a; Li et al., 2011) and have demonstrated that such aptamers can be internalized into cells and carry cargoes into them. Therefore this approach holds much promise for therapeutic development but unfortunately its utility is still hindered by significant limitations (Dollins et al., 2008b; Tiemann and Rossi, 2009; Zhou and Rossi, 2010). Currently, each aptamer can only bind a specific subset of cancer cells based on the biomarkers expressed on the cell surface. Additionally, such aptamers appear to usually internalize into the cells through receptor-mediated endocytosis, which greatly limits their efficacy because their escape from the endosomal compartment

\footnotetext{
${ }^{1}$ Departments of Surgery and Molecular Genetics and Microbiology, Duke University Medical Center, Durham, North Carolina.

${ }^{2}$ Division of Molecular Pharmaceutics, Eshelman School of Pharmacy, University of North Carolina, Chapel Hill, North Carolina.
} 
appears to be inefficient. Thus, in these studies we began to explore whether an aptamer could be identified that can circumvent these problems by binding to and internalizing into multiple cancer cell types and escape the endosomal pathway.

Nucleolin, a protein traditionally found in the nucleolus, where it regulates ribosome biogenesis (Ginisty et al., 1998; Ginisty et al., 1999) and binds telomeres (Pollice et al., 2000), has emerged as a unique biomarker found on the cell surface of rapidly proliferating tumor cells (Hovanessian et al., 2010). Cell surface nucleolin has a short half-life and traffics from the cell surface to the nucleus through a nontraditional mechanism (Huang et al., 2006; Hovanessian et al., 2010). AS1411, a G-quartet DNA aptamer (Girvan et al., 2006; Ireson and Kelland, 2006), has been shown to selectively bind to nucleolin (Teng et al., 2007; Soundararajan et al., 2008) and internalize into a variety of cancer cell lines including renal, breast, and other adenocarcinoma cell lines (Bates et al., 2009a; Bates et al., 2009b; Reyes-Reyes et al., 2010). Because of these properties, we hypothesized that an aptamer chimera engineered with the AS1411 nucleolin aptamer might have potential advantages over other aptamer chimeras. Here we examined the cellular localization of the nucleolin aptamer and chimeras, showing that the aptamer and chimeras localize to the nucleus, a unique property compared to previously described aptamers. We began to examine the mechanism responsible for nuclear localization to determine whether the nucleolin aptamer internalized through the receptor-mediated endocytosis pathway, as well as evaluate the aptamer's ability to deliver therapeutic agents that would take advantage of the nuclear localization of aptamer-based chimeras.

We engineered aptamer-based chimeras to contain spliceswitching oligonucleotides (SSOs) as cargoes. SSOs are a form of antisense technology, where single-stranded oligonucleotides bind to a splice site or splicing enhancer, blocking access to the endogenous splicing machinery, causing an alternate version of a mature mRNA to be generated and translated (Kang et al., 1998; Kole et al., 2004a; Kole et al., 2004b; Ming et al., 2010). SSOs are an emerging tool for generating phenotypic changes in cells (Kang et al., 1998; Bauman et al., 2009; Wan et al., 2009; Ming et al., 2010), and are easy to append to aptamers because they usually contain 2'O-Me modified bases and a phosphorothioate backbone that render the molecules nuclease resistant (Kang et al., 1998). However, to enhance the potential therapeutic utility of SSOs it would be useful to target and efficiently deliver them to the nuclei of specific cells (Lundin et al., 2008; Bauman et al., 2009; Wan et al., 2009; Alam et al., 2010). The nucleolin aptamer-SSO chimera described here effectively delivered SSOs to the nuclei of cells and enhanced splice correction.

\section{Materials and Methods}

\section{RNA and DNA oligonucleotides}

Fluorescently tagged DNA aptamers were ordered from IDT. The splice-switching oligonucleotides and chimeras were prepared according to the method previously reported (Alam et al., 2008).

\section{Oligonucleotide sequences}

AS1411: 5' - GGTGGTGGTGGTTGTGGTGGTGGTGG-3' Control: 5' - CСТССТССТССТTСТССТССТССТСС-3'
EGFR aptamer: 5'- GGCGCUCCGACCUUAGUCUCUGUG CCGCUAUAAUGCAC GGAUUUAAUCGCCGUAGAA AAGCAUGUCAAAGCCGGAACCGUGUAGCACAGC AGA-3'

Luciferase splice-switching oligo: 5'- GUUAUUCUUUAG AAUGGUGC-3'

Control splice-switching oligo: 5'- AUGGCCUCGACGUGC GCGCU-3'

AS1411-Luciferase SSO chimera: 5'- TGGTGGTGGTGGTT GTGGTGGTGGTGGAAA GUUAUUCUUUAGAAUGGU GC-3'

All splice-switching oligos have a 2'-O-Me phosphorothioate chemistry backbone.

\section{siRNA transfections}

All siRNAs were ordered from Dharmacon. Transfections were performed with Lipofectamine 2000 (Invitrogen: 11668019) according to manufacturer's protocol with siRNA at specified concentrations.

\section{Cell culture}

All cells were maintained at $37^{\circ} \mathrm{C}$ and $5 \% \mathrm{CO}_{2}$ in appropriate growth media: HeLa, 786-0 renal adenocarcinoma (ATCC: CRL1932), PC-3 prostate adenocarcinoma (ATCC: CRL1435) and Panc-1 pancreatic epithelioid carcinoma (ATCC: CRL1496), human pancreatic ductal epithelial cells, primary prostate epithelial cells (Lonza: CC-255), and human renal proximal tubule epithelial cells (Lonza: CC-2553)

\section{Cellular uptake and localization}

Non-confocal microscopy was performed on a Zeiss Axio Imager widefield fluorescence microscope with $63 \times$ oil immersion objectives. Cells were seeded and grown on cover slips in multiwell plates for 24 hours before staining.

Aptamer staining. Depending on the assay, aptamers with Alexa 488 or Cy5 conjugated to the $5^{\prime}$ end were ordered from IDT. Fluorescently labeled aptamer was added directly to cell growth media at a concentration of $400 \mathrm{nM}$ and incubated at $37^{\circ} \mathrm{C}$ and $5 \% \mathrm{CO}_{2}$ for 1 hour. Cells were washed 3 in Dulbecco's phosphate-buffered saline (DPBS), then fixed in $4 \%$ paraformaldehyde and washed again $3 \times$ in DPBS.

Antibody staining. Cells were incubated at $37^{\circ} \mathrm{C}$ for 1 hour with rabbit anti-nucleolin immunoglobulin G (IgG) antibody (Abcam) at a concentration of $(1 \mu \mathrm{g} / \mathrm{mL})$. Cells were then washed $3 \times$ in DPBS, then incubated at $37^{\circ} \mathrm{C}$ for 1 hour with a Cy5 conjugated, goat anti-rabbit IgG antibody (GE Healthcare Lifesciences: PA45011) at a dilution of (1:1000). Cells were washed $3 \times$ in DPBS.

For all experiments, cells were then counterstained with 4',6-diamidino-2-phenylindole (DAPI) (Invtirogen: D3571) and mounted to slides with fluoromount-G (Southern Biotech: 0100-01).

Flow cytometry. Cells were incubated in a 12-well plate with AS1411 (AF488) or control aptamer (AF488) at $100 \mathrm{nM}$ for 3 hours $\left(37^{\circ} \mathrm{C}, 5 \% \mathrm{CO}_{2}\right)$ then treated with DNase for 10 minutes to degrade any noninternalized aptamer. Cells were washed with PBS and trypsinized with $0.05 \%$ Trypsin for 
fluorescence-activated cell sorting (FACs) analysis (Becton Dickinson FACSCalibur flow cytometer).

\section{Internalization assays}

HeLa cells in a 24-well plate were incubated with the following drugs: chlorpromazine $\left(7.5 \mu \mathrm{g} / \mathrm{mL}, 2\right.$ hours at $\left.37^{\circ} \mathrm{C}\right)$, amiloride $\left(1 \mathrm{mM}, 2\right.$ hours at $\left.37^{\circ} \mathrm{C}\right)$, and genistein $(150 \mu \mathrm{M}, 2$ hours at $37^{\circ} \mathrm{C}$ ) then washed with Dulbecco's modified Eagle medium $/ 10 \%$ fetal bovine serum (FBS) media. HeLa cells were incubated with $400 \mathrm{nM}$ AS1411 aptamer (AF488) or $400 \mathrm{nM}$ control aptamers (AF488) for 3 hours. HeLa cells were incubated with Alexa 488-transferrin for 30 minutes $\left(10 \mu \mathrm{g} / \mathrm{mL}\right.$ at $\left.37^{\circ} \mathrm{C}\right)$. Cells were washed with PBS $/ 4 \%$ FBS and trypsinized with $0.05 \%$ Trypsin for FACs analysis (Becton Dickinson FACSCalibur flow cytometer).

\section{RNAi assays against nucleolin}

Cells were plated to $50 \%$ confluence 24 hours before treatment with siRNA. The cells were transfected with $50 \mu \mathrm{M}$ siRNA against nucleolin and incubated for 48 hours $\left(37^{\circ} \mathrm{C}\right.$ and $5 \% \mathrm{CO}_{2}$ ) then collected by trypsinization or prepared for cell staining according to the method described above. Trypsinized cells were then separated into fractions using a subcellular protein fractionation kit (Thermo Scientific: 78840). Fractions were then run on $4 \%-20 \%$ polyacrylamide Ready Gel (BioRad: 161-1237) and transferred to a polyvinylidene difluoride membrane. The membrane was probed with rabbit anti-nucleolin IgG antibody (Abcam) 1:1000 then probed with a Cy5 conjugated, goat anti-rabbit antibody 1:1000 (GE Healthcare Lifesciences: PA45011) secondary antibody. The blot was then analyzed on a Typhoon 9410 variable mode imager (GE Healthcare Lifesciences) and quantified.

\section{Oligonucleotide treatment and luciferase assay}

Varying amounts of the Cy5-aptamer-luciferase SSO and free Cy5-luciferase SSO were added to the OPTI-MEM media to give increasing total substrate concentrations. Luciferase induction was determined in the human prostate cancer (PC3)/Luc705 cells over a 24-hour treatment followed by a 2day culture. The luciferase activity was determined using a Luciferase Assay Kit (Promega). Luciferase assay was performed on a FLUOstar Omega microplate reader (BMG Labtech). Protein content was determined by Bradford protein assay (Pierce) with bovine serum albumin as a standard. Background luciferase expression was determined by measuring luciferase activity in the cells without the oligonucleotide treatment, and these values were then subtracted from the results in the treated cells to obtain response values and the final dynamic data. Calculation of the percentage of transcripts switched was performed as previously described (Kang et al., 1998).

\section{Confocal fluorescence microscopy}

Intracellular distribution of the oligonucleotide in living cells was examined using a Zeiss 510 Meta Inverted Laser Scanning Confocal Microscope with $63 \times$ oil immersion objectives. PC3/Luc705 cells were plated in 35-mm glassbottom microwell dishes (MatTek). After transfection of dynamin DN plasmid, intracellular uptake of the oligonucleotide or of Alexa-594-transferrin (Molecular Probes) as a marker for clathrin-coated vesicles was visualized by confocal microscopy.

\section{Data analysis}

Data are expressed as mean \pm standard deviation from 3 measurements unless otherwise noted. Statistical significance was evaluated using $t$-test. The data were analyzed with GraphPad Prism 5 (GraphPad Software, Inc.).

\section{Results}

\section{Differential cellular localization of nucleolin and epithelial growth factor receptor aptamers}

We and others have reported the evaluation of aptameroligonucleotide chimeras. These chimeras have been shown to internalize through a receptor-mediated endocytosis mechanism that leads to endosomal and cytoplasmic localization of the chimeras (McNamara et al., 2006; Dassie et al., 2009; Li et al., 2010). To determine if the nucleolin aptamer localized to a different compartment within treated cells than previously identified aptamers, we performed internalization studies using fluorescently labeled aptamers and fluorescent microscopy. Pancreatic cancer cells, Panc-1, were simultaneously treated with the nucleolin aptamer AS1411 and an aptamer that binds epithelial growth factor receptor (EGFR) (Li et al., 2011). The nucleolin aptamer was conjugated to the red fluorescent dye Cy5 and the EGFR aptamer was conjugated to the green fluorescent dye Alexa488. As shown in Fig. 1A, the EGFR aptamer localized to the cytoplasm and did not enter the nuclei of the Panc-1 cells. By contrast, the nucleolin aptamer localized primarily to the nuclei and nucleoli of the Panc-1 cells with a lower percentage localizing to the cytoplasm (Fig. 1B). When we overlaid the images of the two aptamers, the differences in their localization became even more apparent, especially when the DAPI-stained nuclei were highlighted (Fig. 1C, D). Thus a significant fraction of the nucleolin aptamer localizes to the nuclei of Panc-1 cells and as such shows a distinct subcellular localization compared to other aptamers such as the EGFR aptamer, which predominately localize to the endosomal and cytoplasmic compartments.

\section{Selectivity of the nucleolin aptamer}

To confirm that the internalization and nuclear localization of the nucleolin aptamer in Panc-1 cells is dependent upon nucleolin, we performed siRNA knockdown studies. Panc-1 cells were treated with siRNA against nucleolin mRNA then analyzed for nucleolin aptamer internalization and localization. As shown in Supplementary Fig. S1A, the nucleolin aptamer internalizes and localizes to the nuclei of Panc-1 cells treated with a control siRNA (Supplementary Data are available online at www.liebertpub.com/nat). However, siRNA-mediated knockdown of nucleolin expression greatly reduced such internalization and localization (Supplementary Fig. S1B). To confirm that cell surface expression of nucleolin was knocked down in the Panc-1 cells treated with the siRNA against nucleolin, we stained the treated cells with an antinucleolin antibody (Supplementary Fig. 1C, D). These studies confirmed the effectiveness of the siRNA for knocking down cell surface expression of nucleolin and demonstrated that 
FIG. 1. The nucleolin and epithelial growth factor receptor (EGFR) aptamers show differential cellular localization. Panc-1 cells were grown to near confluence on coverslips and then incubated with Cy5-AS1411 and Alexa488-EGFR aptamers at a concentration of $100 \mathrm{nM}$ each for 1hour. Cells were then fixed, stained with DAPI, and analyzed by fluorescent microscopy. (A) The Alexa488-EGFR aptamer. (B) The Cy5-AS1411 aptamer. (C) An overlay image of the 2 aptamers. (D) An overlay image of the 2 aptamers with nuclei stained with DAPI.
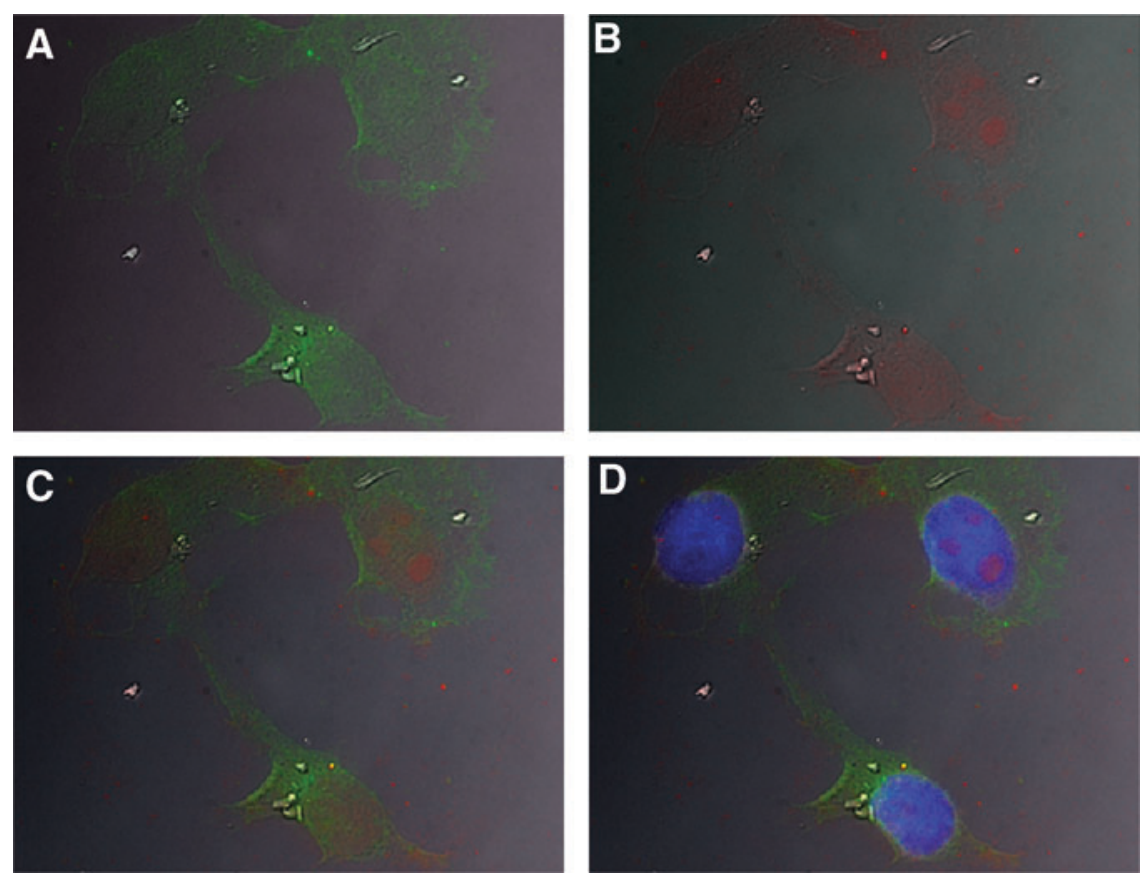

nucleolin is required for the internalization and nuclear localization of the aptamer in Panc- 1 cells.

Next we investigated nucleolin aptamer uptake in a variety of cancer cell types and corresponding noncancerous cell types. Using Western blot analysis, we observed that a much higher level of nucleolin is expressed on the surface of cancer cells compared with their corresponding noncancerous cell types (Supplementary Fig. S2A). Cells were then analyzed to determine whether the difference in surface nucleolin expression would lead to differences in nucleolin aptamer binding and uptake using flow cytometry and fluorescent microscopy. As shown in Supplementary Fig. 2, the nucleolin aptamer bound and internalized into various cancer cell lines (Supplementary Fig. 2B-D and unpublished results), and not the corresponding noncancerous cell types (Supplementary Fig. 2E, and unpublished results). A labeled control aptamer did not bind or internalize into any of the cell types, demonstrating the specificity of the aptamer.

\section{Mechanism of internalization}

The observation that the nucleolin aptamer localized to the nucleus and the EGFR aptamer to the cytoplasm suggested that the nucleolin aptamer was internalizing through a distinct mechanism compared with other aptamers tested to date. However, cell surface nucleolin has not been well characterized, and its mechanism of internalization remains largely unknown, though recent publications suggest that
A

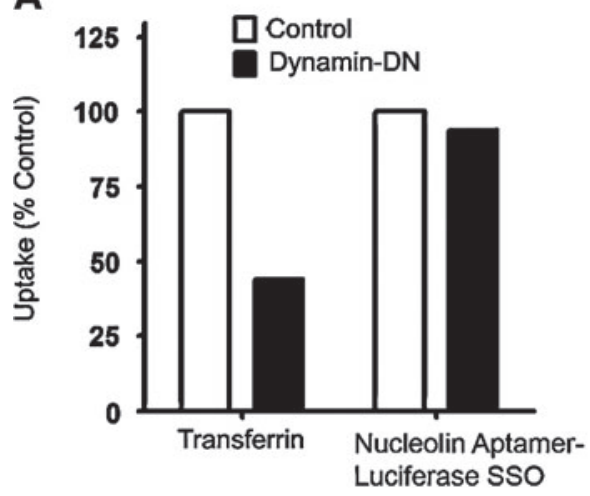

B
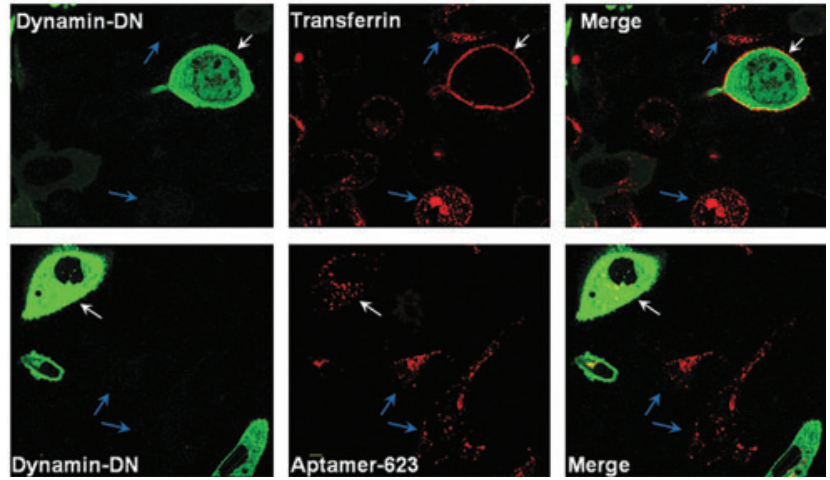

FIG. 2. The nucleolin aptamer internalizes through a dynamin-independent mechanism. (A) PC-3/Luc 705 cells were grown to near confluence and then transfected with a dynamin-DN/GFP encoding plasmid. Alexa-594 labeled aptamer was added to cells, and intracellular uptake of the nucleolin aptamer or of Alexa-594-labeled transferrin was analyzed and quantified. PC-3/Luc 705 cells that did not contain the dynamin-DN/GFP plasmid were used as the control for the contribution of dynamin. (B) A representative confocal microscopy image of the data from panel A: from the left, the first panel shows the cells transfected with the dynamin-DN/GFP plasmid in green, the second panel shows the location of either transferrin (top) or aptamer (bottom), and the third panel is a merged overlay of the first 2. White arrows indicate GFP/ dynamin-DN positive cells, and blue arrows indicate GFP/dynamin-DN negative cells. GFP, green fluorescent protein. 
nucleolin may associate with a myosin motor that traffics along actin filaments (Huang et al., 2006). To begin to determine the mechanism of the nucleolin aptamer's internalization, we initially evaluated whether the aptamer internalization was dependent upon dynamin using a dominant negative mutant version of the protein. Dynamins are a class of motor proteins responsible for the scission of newly formed vesicles, playing a large role in endocytosis (Vallee et al., 1993); therefore, in cells lacking dynamin, receptormediated endocytosis was inhibited. As shown in Fig. 2, the nucleolin aptamer internalized into PC3 cells expressing a dominant negative mutant version of dynamin (dynaminDN) as well as it did into wild-type PC3 cells (Fig. 2A). By contrast, transferrin, a cell surface receptor that internalizes through endocytosis, was greatly inhibited in the dynaminDN expressing cells compared with cells not expressing dynamin-DN (Fig. 2A). Cells from these internalization studies were further examined through fluorescent microscopy. PC3 cells expressing the dominant negative dynamin, labeled with green fluorescent protein (GFP), were treated with a Cy5 conjugated nucleolin aptamer. As shown in Fig. 2B (bottom), the aptamer internalized into the cytoplasm and nucleus of the dynamin-DN cells, indicating that the aptamer internalized through a dynamin-independent mechanism. In dynamin-DN/GFP expressing cells, internalization of Alexa594-transferrin was assessed. Transferrin was not able to internalize into the dynamin-DN expressing cells and was seen associated with only the outer membrane of the dynamin-DN cells (Fig.2B, top, white arrows). However, transferrin was able to internalize into cells lacking the dominant negative mutant protein as noted by the blue arrows (Fig. 2B, top). Thus the nucleolin aptamer enters PC-3 cells through a dynamin-independent mechanism.

To further evaluate the mechanism of nucleolin aptamer internalization, we employed a panel of internalization inhibitors and HeLa cells. As shown in Fig. 3A, the nucleolin aptamer rapidly internalizes and localizes to the nuclei of HeLa cells. Next we tested 3 uptake inhibitors to determine if they could inhibit aptamer uptake in HeLa cells. Amiloride (AML) lowers submembranous $\mathrm{pH}$, which reduces actin remodeling at the cell membrane (Mercer and Helenius, 2009; Mercer et al., 2010). AML has traditionally been used as an inhibitor of macropinocytosis but has been shown to block some clathrin-mediated endocytosis and internalization through lipid rafts (Ivanov et al., 2004; Wadia et al., 2004; Mercer and Helenius, 2009; Koivusalo et al., 2010; Mercer et al., 2010) (Fig. 3B). Chlorpromazine (CPZ) prevents the recycling of clathrin, which inhibits clathrin-mediated endocytosis, a form of receptor-mediated endocytosis in which small molecules aggregate around an invagination and close a vesicle into the cell (Sieczkarski and Whittaker, 2002) (Fig. 3B). Genistein (GEN) inhibited caveolar endocytosis, which is a form of clathrin-independent endocytosis caused by invagination of lipid rafts (Parton et al., 1994; Sieczkarski and Whittaker, 2002) (Fig. 3B). The uptake of the nucleolin aptamer was moderate but significantly inhibited by both $\mathrm{CPZ}$ and AML, but neither totally prevented aptamer internalization (Fig. 3C). GEN had no effect on either transferrin or aptamer uptake, which indicated that neither caveolar endocytosis nor lipid-raft-mediated endocytosis were the mechanisms of internalization (Fig. 3C). Transferrin, a wellcharacterized cell surface receptor, served as a positive control throughout these uptake studies, and cells treated with dimethyl sulfoxide (DMSO) served as negative control because all uptake inhibitors were dissolved in DMSO. As expected, transferrin was inhibited by CPZ, but it was also inhibited somewhat by AML, which may have been caused by the toxicity of AML or its effects on the clathrin-mediated endocytosis pathway (Fig. 3C). The mechanism of nucleolin aptamer internalization and nuclear localization cannot be explained by the standard model of receptor-mediated endocytosis or macropinocytosis alone and requires further investigation. However, we observed that the aptamer did not internalize through receptor-mediated endocytosis, which is the accepted mechanism of uptake for other aptamers (eg, the PSMA and EGFR aptamers). These data demonstrate that the nucleolin aptamer internalizes through a nonstandard mechanism, which allows for nuclear localization and the apparent ability to escape the endosomal compartment.

\section{Efficacy of nucleolin aptamer-SSO Chimeras}

Because the nucleolin aptamer selectively localizes to the nuclei of cancer cells and efficiently internalizes into PC 3 cells, we investigated whether the nucleolin aptamer could be utilized to deliver SSOs to the nucleus of PC3 cells containing a splice-switching luciferase report construct (PC3/Luc 705 cells). We examined whether an aptamer-SSO chimera could deliver the SSO to the nucleus and increase the efficacy of SSO activity. In our proof-of-principle experiments we employed PC3/Luc 705 cells, a prostate cancer cell type containing a luciferase reporter construct with a premature stop codon, which prevents full-length luciferase from being translated. When treated by an SSO, the splicing of the pre-mRNA derived from the luciferase gene is altered to exclude the stop codon, allowing the repaired luciferase mRNA to be translated and luciferase to be produced (Ming et al., 2010). PC3/ Luc 705 cells were incubated with various oligonucleotides without transfection agent to determine if the aptamerluciferase SSO chimera could alter splicing and enhance luciferase production. As shown in Fig. 4A, the aptamer-SSO chimera significantly increased luciferase production compared with untreated cells, cells treated with a control SSO, a mutant aptamer-SSO chimera, and the SSO alone. To assess the effect of the oligonucleotide treatments on pre-mRNA splicing, we examined the ratio of mutant versus repaired RNA produced. Total RNA was isolated from treated cells and quantitative competitive reverse transcription-polymerase chain reaction (qCRT-PCR) was performed with primers to differentiate between the 2 versions of the luciferase mRNA (Kang et al., 1998). The products of the qcRT-PCR were separated on a gel and quantified. As shown in Fig. 4B, the aptamer-luciferase SSO chimera increased the percentage of repaired mRNA by $13 \%$ over the baseline $10 \%$ found in untreated cells and cells treated with a mutant aptamer-SSO. Moreover, even though the nucleolin aptamer-SSO chimera is much longer than the luciferase SSO alone (50 nt vs. $20 \mathrm{nt}$ ), the chimera was still more efficient than the SSO alone (Fig. 4B). These results demonstrated that the aptamer-SSOs can alter pre-mRNA splicing in PC-3 prostate cancer cells.

To further examine the efficiency of the aptamer-luciferase SSO chimera compared to the SSO alone, we treated PC3/Luc 705 cells with various concentrations of either the aptamerSSO chimera or the SSO alone. At a concentration of $100 \mathrm{nM}$, 
A

FIG. 3. Evaluating aptamer internalization with uptake inhibitors. (A) HeLa cells were grown to $\sim 60 \%$ confluence on coverslips and then incubated with Alexa488-nucleolin aptamer or Alexa488control aptamer at a concentration of $100 \mathrm{nM}$ for 1 hour. Cells were then fixed, stained with DAPI, and analyzed by fluorescent microscopy. (B) The clathrinmediated pathway was inhibited by chlorpromazine (CPZ). The caveloarmediated and lipid raft-mediated pathways are inhibited by genistein (GEN). The macropinocytosis pathway is inhibited by amiloride (AML). To some extent, AML also inhibits other pathways that require actin synthesis at the cell membrane. The dynamin-DN mutant protein prevents dynamin dependent pathways. (Adapted from Mercer, J. and Helenius, 2009.) (C) HeLa cells were grown to near confluence then incubated with AML $\left(1 \mathrm{mM}, 2\right.$ hours at $\left.37^{\circ} \mathrm{C}\right)$, $\mathrm{CPZ}\left(7.5 \mu \mathrm{g} / \mathrm{mL}, 2\right.$ hours at $\left.37^{\circ} \mathrm{C}\right)$, or GEN $\left(150 \mu \mathrm{M}, 2\right.$ hours at $\left.37^{\circ} \mathrm{C}\right)$, then washed. The 3 inhibitors were solubilized in dimethyl sulfoxide (DMSO); therefore, the untreated-control group was treated with an equal amount of DMSO. Cells were incubated with Alexa488-nucleolin aptamer or Alexa488-control aptamer for 3 hours at a concentration of $400 \mathrm{nM}$. The cells were treated with DNase to degrade any aptamer on the cell surface that had not been endocytosed then washed, evaluated by flow cytometry, and quantified (statistical analyses were performed against the nucleolin aptamer treated DMSO control $* * * P=0.0051$, **P $\left.=0.0451, \quad{ }^{*} P=0.1353\right)$. Uptake of Alexa-488-transferrin was evaluated as well.

B

C

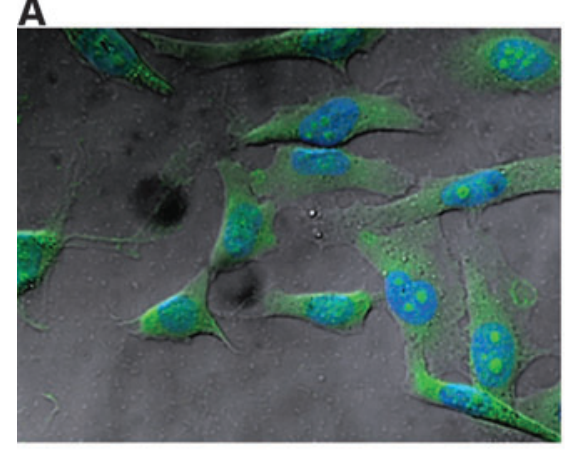

Nucleolin Aptamer
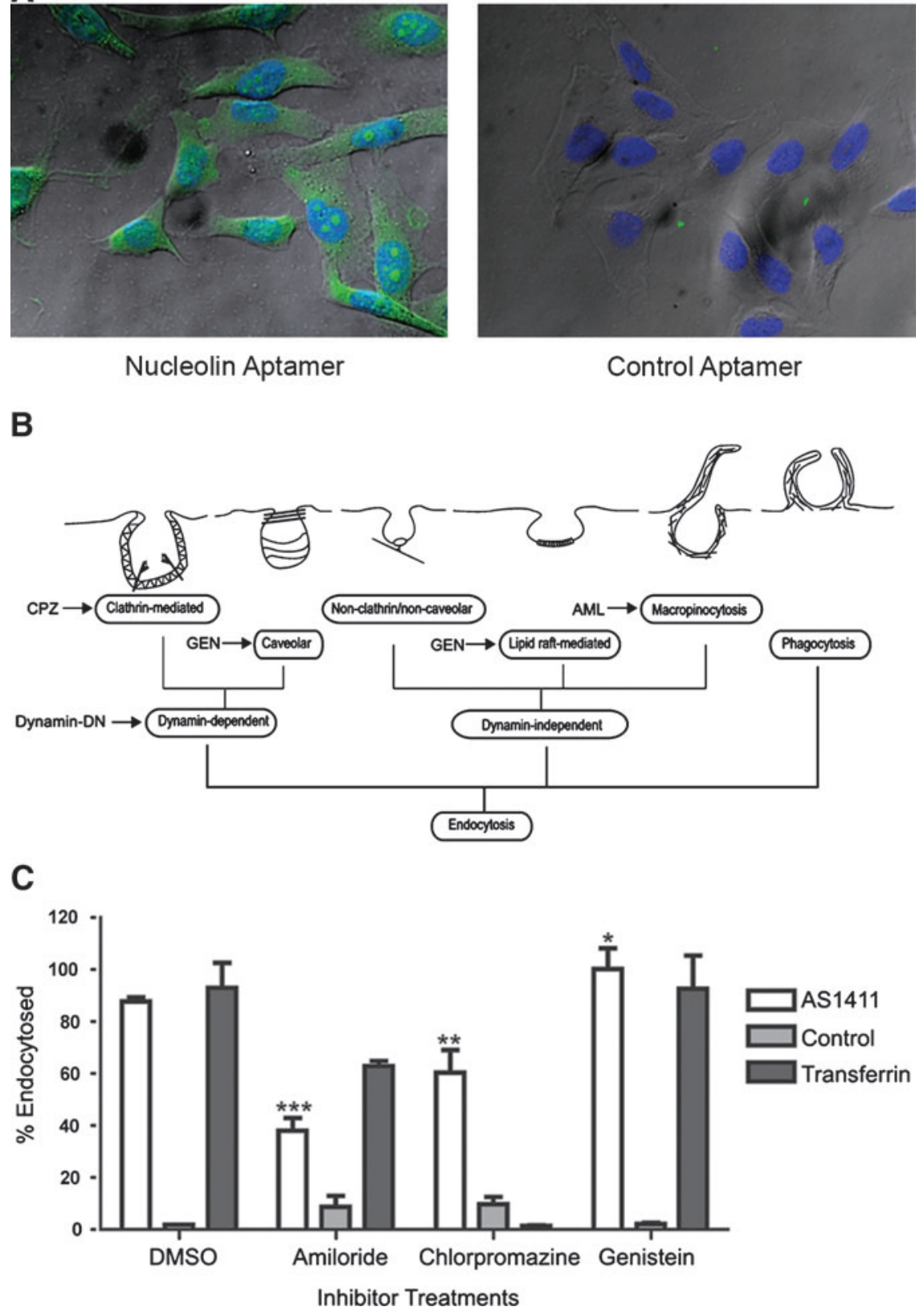

Control Aptamer the amount of luciferase correction generated by the SSO alone began to plateau and produce a maximal amount of protein. This amount of luciferase was produced in cells treated with $\sim 20 \mathrm{nM}$ of the aptamer-luciferase SSO chimera and higher levels of aptamer-SSO produced higher levels of luciferase activity than achieved by the SSO alone (Fig. 5). Thus the aptamer-SSO is approximately 5-fold more potent than the SSO alone.

\section{Discussion}

Our results demonstrate that the nucleolin aptamer can be utilized to deliver oligonucleotides to the nucleus of cancerous cells and alter nuclear events such as pre-mRNA splicing. The nucleolin aptamer bound to cell-membrane-associated nucleolin and internalized through a novel mechanism that 


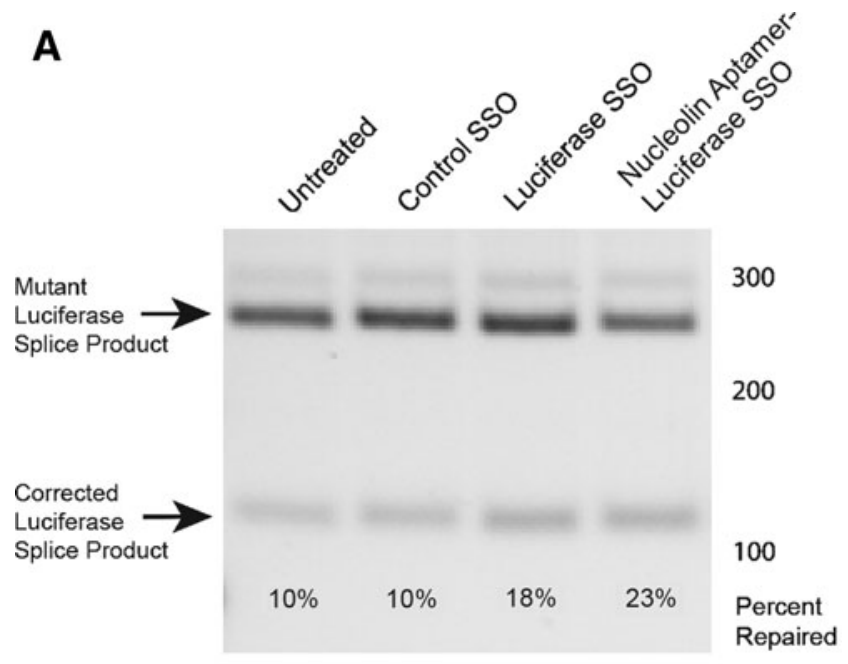

B

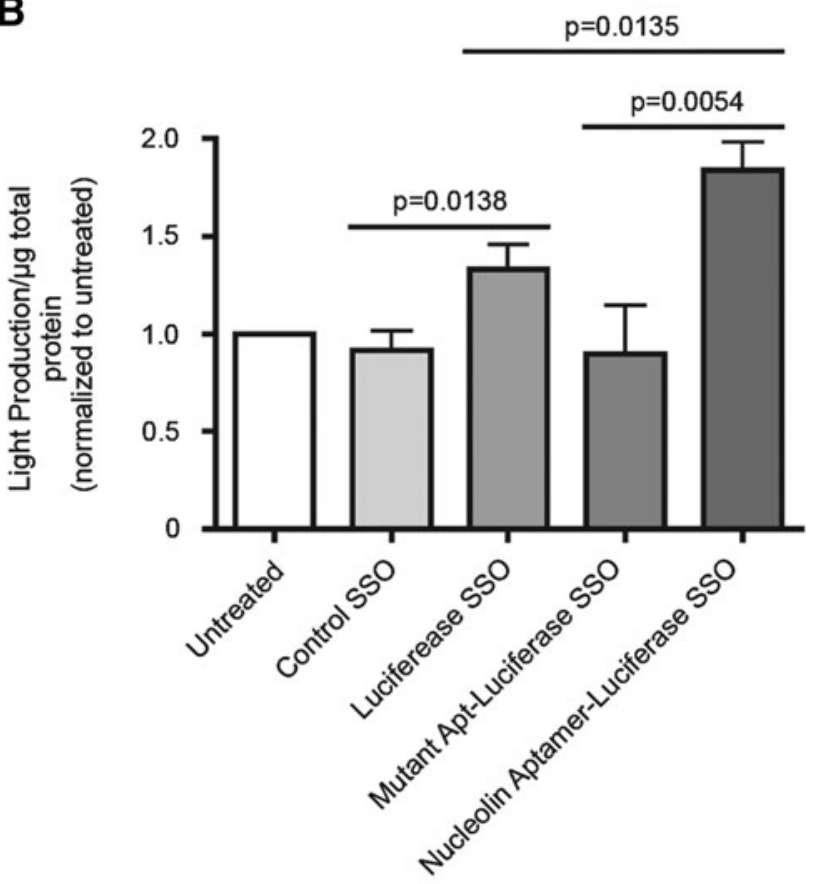

FIG. 4. Aptamer-splice-switching oligonucleotide (SSO) chimeras alter pre-RNA splicing and induce luciferase production. PC-3/Luc 705 cells were grown to $\sim 60 \%$ confluence then incubated for 48 hours with the various oligonucleotides indicated. (A) RNA was isolated from cells and qcRTPCR was performed to examine the ratio of unswitched mutant (longer product) to switched or repaired RNA (shorter product) present. The numbers indicate the percent of mRNA repaired by each treatment. Untreated cells show a background level of repaired luciferase mRNA of $10 \%$. (B) Cells were incubated with the various oligonucleotides $(100 \mathrm{nM})$ indicated without transfection reagents. A microplate reader analyzed cell lysates for light emission, and total protein was determined by Bradford assay. Data was normalized to the untreated cell samples. P-values are shown between the various treatment groups. qcRT-PCR, quantitative competitive reverse transcription-polymerase chain reaction.

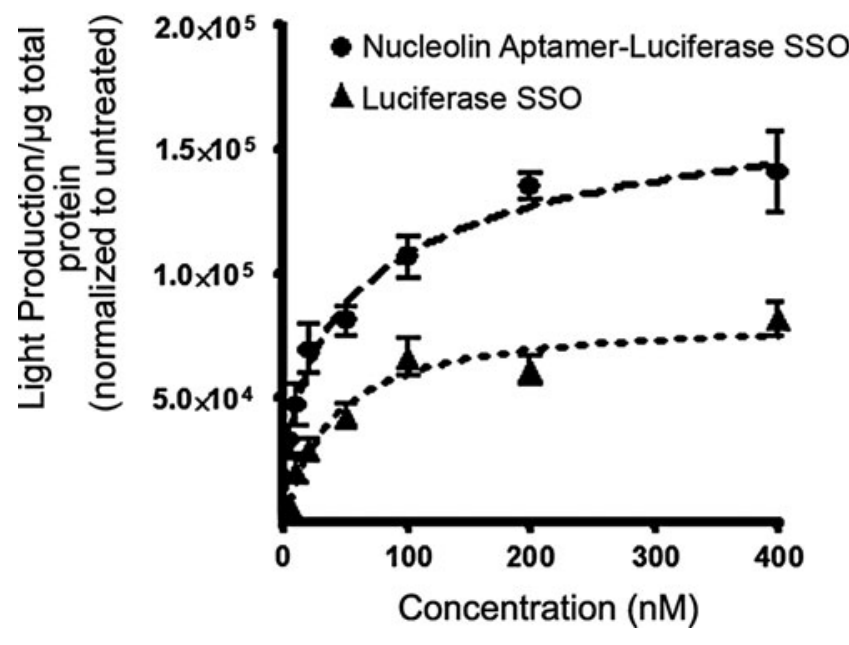

FIG. 5. Dose response comparing the effects of nucleolin aptamer-SSO chimera and the SSO alone. PC-3/Luc 705 cells were grown to $\sim 60 \%$ confluence then incubated for 48 hours with various concentrations of the nucleolin aptamer-luciferase SSO chimera or the luciferase SSO alone. A microplate reader analyzed cell lysates for light emission, and total protein was determined by Bradford assay.

of SSO-based therapies is that only a small percentage of premRNA has to be repaired to generate a phenotypic effect. We observed that the nucleolin aptamer-luciferase SSO increased proper splicing of luciferase by $13 \%$ (Fig. 4), a level of repair that would be expected to result in correction of the phenotypes associated with many genetic mutations. For example, encouraging studies from the Kole lab indicate that only a small percentage of Bcl-xL needed to be "switched" to Bcl-xS by SSOs in order to induce apoptosis (Zhang et al., 2007; Bauman et al., 2010; Bauman and Kole, 2011). However, no effective method for selective delivery of proapoptotic SSOs to cancer cells has been described. Our lab and others have shown that aptamer chimeras possess the properties necessary for in vivo targeting of oligonucleotides (McNamara et al., 2006; Dassie et al., 2009) and the work described herein demonstrates for the first time that an aptamer can selectively deliver a cargo to the nucleus. Thus we believe that by appending therapeutic SSOs to the nucleolin aptamer such as the one described for Bcl-xL $(42,44)$, such aptamer-SSO chimeras may be particularly useful at delivering SSOs to the nuclei of cancerous cells and selectively inducing apoptosis. Within the nucleus, the aptamer chimera appears to further concentrate in the nucleolus, which is likely because nucleolin is more concentrated in the heterochromatin-rich nucleolus. Nevertheless, the splice-switching oligonucleotide is still free to interact with nuclear pre-mRNA and alter impact splicing. However, such aptamer chimeras may be particularly well suited for targeting nucleolar events such as sequestering genes into heterochromatin or ribosome biogenesis. In addition, it may be possible to further optimize the potency of aptamer-SSO chimeras for modulating pre-mRNA splicing by engineering chimeras that release their SSO once they reach the nucleus. Moreover, other types of oligonucleotidebased therapies such as aptamers against intracellular or nuclear targets and other antisense agents such as LNAs can likely be delivered to the nuclei of cancer cells with the nucleolin aptamer. 
The use of aptamers as a delivery agent is still a relatively new field. However, several aptamer chimeras have now shown promise to selectively deliver therapeutics to targeted cancer cells (McNamara et al., 2006; Dassie et al., 2009; Zhou and Rossi, 2010). In principle, this approach can reduce the adverse effects associated with therapies and potentially reduce the cost of the therapeutic approach. Our results demonstrate that aptamer chimeras can enter through a mechanism that avoids or escapes the endosomal compartment and localizes to the nucleus, and the nucleolus, which opens new opportunities for delivery of therapeutic agents that target nuclear events. We believe that the results of our study have laid the foundation for future work that will demonstrate the therapeutic value of aptamer chimeras and nuclear delivery.

\section{Acknowledgments}

This research was supported in part by an NIH T32 Postdoctoral Fellowship (EDP) and an NIH Grant R01CA129190 (BAS).

\section{Author Disclosure Statement}

No competing financial interests exist.

\section{References}

ALAM, M., MING, X., DIXIT, V., FISHER, M., CHEN, X., and JULIANO, R. (2010). The biological effect of an antisense oligonucleotide depends on its route of endocytosis and trafficking. Oligonucleotides 20, 103-109.

BATES, P., CHOI, E., and NAYAK, L. (2009a). G-rich oligonucleotides for cancer treatment. Methods Mol. Biol. 542, 379-392.

BATES, P.J., LABER, D.A., MILLER, D.M., THOMAS, S.D., and TRENT, J.O. (2009b). Discovery and development of the Grich oligonucleotide AS1411 as a novel treatment for cancer. Exp. Mol. Pathol. 86, 151-164.

BAUMAN, J., JEARAWIRIYAPAISARN, N., and KOLE, R. (2009). Therapeutic potential of splice-switching oligonucleotides. Oligonucleotides 19, 1-13.

BAUMAN, J.A., and KOLE, R. (2011). Modulation of RNA splicing as a potential treatment for cancer. Bioeng. Bugs 2, $125-128$

BAUMAN, J.A., LI, S.D., YANG, A., HUANG, L., and KOLE, R. (2010). Anti-tumor activity of splice-switching oligonucleotides. Nucleic Acids Res. 38, 8348-8356.

CHU, T., MARKS, J.R., LAVERY, L., FAULKNER, S., ROSENBLUM, M., ELLINGTON, A., and LEVY, M. (2006a). Aptamer:toxin conjugates that specifically target prostate tumor cells. Cancer Res. 66, 5989-5992.

CHU, T., TWU, K., ELLINGTON, A., and LEVY M. (2006b). Aptamer mediated siRNA delivery. Nucleic Acids Res. 34, e73.

DASSIE, J., LIU, X., THOMAS, G., WHITAKER, R., THIEL, K., STOCKDALE, K., MEYERHOLZ, D., MCCAFFREY, A., MCNAMARA, J.N., and GIANGRANDE, P. (2009). Systemic administration of optimized aptamer-siRNA chimeras promotes regression of PSMA-expressing tumors. Nat. Biotechnol. 27, 839-849.

DOLLINS, C., NAIR, S., BOCZKOWSKI, D., LEE, J., LAYZER, J., GILBOA, E., and SULLENGER B. (2008a). Assembling OX40 aptamers on a molecular scaffold to create a receptor-activating aptamer. Chem. Biol. 15, 675-682.
DOLLINS, C., NAIR, S., SULLENGER, B. (2008b). Aptamers in immunotherapy. Hum. Gene Ther. 19, 443-450.

FAROKHZAD, O.C., CHENG, J., TEPLY, B.A., SHERIFI, I., JON, S., KANTOFF, P.W., RICHIE, J.P., and LANGER, R. (2006). Targeted nanoparticle-aptamer bioconjugates for cancer chemotherapy in vivo. Proc. Natl. Acad. Sci. U. S. A. 103, 63156320.

GINISTY, H., AMALRIC, F., and BOUVET, P. (1998). Nucleolin functions in the first step of ribosomal RNA processing. EMBO J 17, 1476-1486.

GINISTY, H., SICARD, H., ROGER, B., and BOUVET, P. (1999). Structure and functions of nucleolin. J. Cell Sci. 112(6), 761-772.

GIRVAN, A., TENG, Y., CASSON, L., THOMAS, S., JÜLIGER, S., BALL, M., KLEIN, J., PIERCE, W.J., BARVE, S., and BATES, P. (2006). AGRO100 inhibits activation of nuclear factorkappaB (NF-kappaB) by forming a complex with NF-kappaB essential modulator (NEMO) and nucleolin. Mol. Cancer Ther. 5, 1790-1799.

HOVANESSIAN, A.G., SOUNDARAMOURTY, C., EL KHOURY, D., NONDIER, I., SVAB, J., and KRUST, B. (2010). Surface expressed nucleolin is constantly induced in tumor cells to mediate calcium-dependent ligand internalization. PLoS One 5, e15787.

HUANG, Y., SHI, H., ZHOU, H., SONG, X., YUAN, S., and LUO, Y. (2006). The angiogenic function of nucleolin is mediated by vascular endothelial growth factor and nonmuscle myosin. Blood 107, 3564-3571.

IRESON, C., and KELLAND, L. (2006). Discovery and development of anticancer aptamers. Mol. Cancer Ther. 5, 2957-2962.

IVANOV, A.I., NUSRAT, A., and PARKOS, C.A. (2004). Endocytosis of epithelial apical junctional proteins by a clathrinmediated pathway into a unique storage compartment. Mol. Biol. Cell 15, 176-188.

KANG, S.H., CHO, M.J., and KOLE, R. (1998). Up-regulation of luciferase gene expression with antisense oligonucleotides: implications and applications in functional assay development. Biochemistry 37, 6235-6239.

KIM, E., JUNG, Y., CHOI, H., YANG, J., SUH, J.S., HUH, Y.M., KIM, K., and HAAM, S. (2010). Prostate cancer cell death produced by the co-delivery of Bcl-xL shRNA and doxorubicin using an aptamer-conjugated polyplex. Biomaterials 31, 4592-9.

KOIVUSALO, M., WELCH, C., HAYASHI, H., SCOTT, C.C., KIM, M., ALEXANDER, T., TOURET, N., HAHN, K.M., and GRINSTEIN, S. (2010). Amiloride inhibits macropinocytosis by lowering submembranous $\mathrm{pH}$ and preventing Rac1 and Cdc42 signaling. J. Cell Biol. 188, 547-563.

KOLE, R., VACEK, M., and WILLIAMS, T. (2004a). Modification of alternative splicing by antisense therapeutics. Oligonucleotides 14, 65-74.

KOLE, R., WILLIAMS, T., and COHEN, L. (2004b). RNA modulation, repair and remodeling by splice switching oligonucleotides. Acta Biochim. Pol. 51, 373-378.

LI, N., LARSON, T., NGUYEN, H., SOKOLOV, K., and ELLINGTON, A. (2010). Directed evolution of gold nanoparticle delivery to cells. Chem. Commun. (Camb.) 46, 392-394.

LI, N., NGUYEN, H.H., BYROM, M., and ELLINGTON, A.D. (2011). Inhibition of cell proliferation by an anti-EGFR aptamer. PLoS One 6, e20299.

LUNDIN, P., JOHANSSON, H., GUTERSTAM, P., HOLM, T., HANSEN, M., LANGEL, U., and EL ANDALOUSSI, S. (2008). Distinct uptake routes of cell-penetrating peptide conjugates. Bioconjug. Chem. 19, 2535-2542. 
MCNAMARA, J.N., ANDRECHEK, E., WANG, Y., VILES, K., REMPEL, R., GILBOA, E., SULLENGER, B., and GIANGRANDE, P. (2006). Cell type-specific delivery of siRNAs with aptamer-siRNA chimeras. Nat Biotechnol 24, 1005-1015.

MERCER, J., and HELENIUS, A. (2009). Virus entry by macropinocytosis. Nat. Cell Biol. 11, 510-520.

MERCER, J., SCHELHAAS, M., and HELENIUS, A. (2010). Virus entry by endocytosis. Annu. Rev. Biochem. 79, 803-833.

MIN, K., JO, H., SONG, K., CHO, M., CHUN, Y.S., JON, S., KIM, W.J., and BAN, C. (2011). Dual-aptamer-based delivery vehicle of doxorubicin to both PSMA (+) and PSMA (-) prostate cancers. Biomaterials 32, 2124-2132.

MING, X., ALAM, M., FISHER, M., YAN, Y., CHEN, X., and JULIANO, R. (2010). Intracellular delivery of an antisense oligonucleotide via endocytosis of a $\mathrm{G}$ protein-coupled receptor. Nucleic Acids Res. 38, 6567-6576.

PARTON, R.G., JOGGERST, B., and SIMONS, K. (1994). Regulated internalization of caveolae. J. Cell Biol. 127, 1199-1215.

POLLICE, A., ZIBELLA, M., BILAUD, T., LAROCHE, T., PULITZER, J., and GILSON, E. (2000). In vitro binding of nucleolin to double-stranded telomeric DNA. Biochem. Biophys. Res. Commun. 268, 909-915.

REYES-REYES, E.M., TENG, Y., and BATES, P.J. (2010). A new paradigm for aptamer therapeutic AS1411 action: uptake by macropinocytosis and its stimulation by a nucleolin-dependent mechanism. Cancer Res. 70, 8617-8629.

SIECZKARSKI, S.B., and WHITTAKER, G.R. (2002). Influenza virus can enter and infect cells in the absence of clathrinmediated endocytosis. J. Virol. 76, 10455-10464.

SOUNDARARAJAN, S., CHEN, W., SPICER, E., COURTENAYLUCK, N., and FERNANDES, D. (2008). The nucleolin targeting aptamer AS1411 destabilizes Bcl-2 messenger RNA in human breast cancer cells. Cancer Res. 68, 2358-2365.

TENG, Y., GIRVAN, A., CASSON, L., PIERCE, W.J., QIAN, M., THOMAS, S., and BATES, P. (2007). AS1411 alters the localization of a complex containing protein arginine methyltransferase 5 and nucleolin. Cancer Res. 67, 10491-10500.

TIEMANN, K., and ROSSI, J. (2009). RNAi-based therapeuticscurrent status, challenges and prospects. EMBO Mol. Med. 1, 142-151.
VALLEE, R.B., HERSKOVITS, J.S., AGHAJANIAN, J.G., BURGESS, C.C., and SHPETNER, H.S. (1993). Dynamin, a GTPase involved in the initial stages of endocytosis. Ciba Found. Symp. 176, 185-193; discussion 193-197.

WADIA, J.S., STAN, R.V., and DOWDY, S.F. (2004). Transducible TAT-HA fusogenic peptide enhances escape of TAT-fusion proteins after lipid raft macropinocytosis. Nat. Med. 10, 310-315.

WAN, J., SAZANI, P., and KOLE, R. (2009). Modification of HER2 pre-mRNA alternative splicing and its effects on breast cancer cells. Int. J. Cancer 124, 772-777.

WU, X., DING, B., GAO, J., WANG, H., FAN, W., WANG, X., ZHANG, W., YE, L., ZHANG, M., DING, X. et al. (2011). Second-generation aptamer-conjugated PSMA-targeted delivery system for prostate cancer therapy. Int. J. Nanomedicine 6, 1747-1756.

WULLNER, U., NEEF, I., ELLER, A., KLEINES, M., TUR, M.K., and BARTH, S. (2008). Cell-specific induction of apoptosis by rationally designed bivalent aptamer-siRNA transcripts silencing eukaryotic elongation factor 2. Curr. Cancer Drug Targets 8, 554-565.

ZHANG, N., PEAIRS, J.J., YANG, P., TYRRELL, J., ROBERTS, J., KOLE, R., and JAFFE, G.J. (2007). The importance of Bcl-xL in the survival of human RPE cells. Invest. Ophthalmol. Vis. Sci. 48, 3846-3853.

ZHOU, J., and ROSSI, J. (2010). Aptamer-targeted cell-specific RNA interference. Silence 1, 4.

$$
\begin{array}{r}
\text { Address correspondence to: } \\
\text { Dr. Bruce A. Sullenger } \\
\text { Department of Surgery } \\
\text { Duke University Medical Center } \\
\text { Box } 103035 \\
\text { Durham, NC } 27710
\end{array}
$$

E-mail: bruce.sullenger@duke.edu

Received for publication March 9, 2012; accepted after revision May 7, 2012. 